\title{
LA-UR- $03-0569$
}

Approved for public release;

distribution is unlimited.

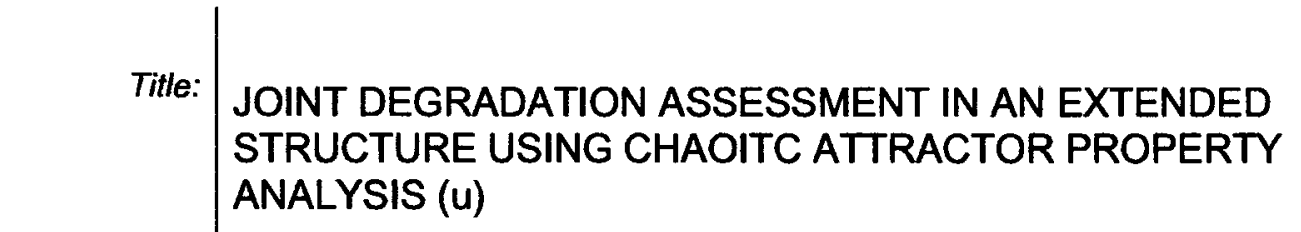

Submitted to:

Jeannette R. Wait, LANL, ESA-WR

Michael D. Todd, US Naval Research Laboratory

Jonathan Nichols, US Naval Research Laboratory

Stephen Trickey, US Naval Research Laboratory

SPIE's 10th Annual International Symposium On Smart

Structures and Materials

San Diego, CA

March 2-6, 2003

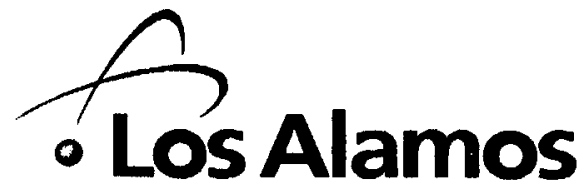

NATIONAL LABORATORY

Los Alamos National Laboratory, an affirmative action/equal opportunity employer, is operated by the University of California for the U.S. Department of Energy under contract W-7405-ENG-36. By acceptance of this article, the publisher recognizes that the U.S. Government retains a nonexclusive, royalty-free license to publish or reproduce the published form of this contribution, or to allow others to do so, for U.S. Government purposes. Los Alamos National Laboratory requests that the publisher identify this article as work performed under the auspices of the U.S. Department of Energy. Los Alamos National Laboratory strongly supports academic freedom and a researcher's right to publish; as an institution, however, the Laboratory does not endorse the viewpoint of a publication or guarantee its technical correctness. 


\title{
Joint degradation assessment in an extended structure using chaotic attractor property analysis
}

\author{
Michael Todd ${ }^{a}$, Jeannette Wait ${ }^{b}$, Jonathan Nichols ${ }^{a}$, and Stephen Trickey ${ }^{a}$ \\ ${ }^{a}$ Code 5673 , U. S. Naval Research Laboratory, Washington, DC 20375 USA \\ ${ }^{b}$ ESA-WR, Los Alamos National Laboratory, MS T006, Los Alamos, NM 87545 USA
}

\begin{abstract}
Recently, a new approach in vibration-based structural health monitoring has been developed utilizing features extracted from concepts in nonlinear dynamics systems theory. The structure is excited with a low-dimensional chaotic input, and the steady-state structural response attractor is reconstructed using a false nearest neighbors algorithm. Certain features have been computed from the attractor such as average local "neighborhood" variance, and these features have been shown in previous works to exceed the damage resolving capability of traditional modal-based features in several computational and experimental studies. In this work, we adopt a similar attractor approach, but we present a feature based on nonlinear predictive models of evolving attractor geometry. This feature has an advantage over previous attractor-based features in that the input excitation need not be monitored. We apply this overall approach to a steel frame model of a multi-story building, where damage is incurred by the loosening of bolted connections between model members.
\end{abstract}

Keywords: structural health monitoring, chaos, damage detection

\section{INTRODUCTION}

Structural owners place a high priority on assessing the functional capability of a structure. Economic constraints, variable mission requirements, and liability considerations urge structural owners towards reducing maintenance and operational costs. Historically, most owners have relied upon inspection techniques and fixed maintenance or rotation schedules for assessing structural health, and little recourse was made to automated methodologies or algorithms. However, with rapid recent advances in structural materials, sensing technologies, and data processing and management capability, owners have increasingly embraced research and development in advancing the state of the art. One prominent example is the United States Navy, who have identified a "Total Ownership Cost Reduction" Future Naval Capability (FNC) that calls for a variety of initiatives aimed at reducing maintenance and operational costs of all Navy structures as the Navy officially adopts a condition basis for structural maintenance.

Much of the technical advancement over the last several decades has led to the field of non-destructive evaluation (NDE). The major approaches have included ultrasonic or acoustic wave propagation, thermal imaging, $\mathrm{X}$-ray radiography, and eddy-current methods; an overall summary may be found in, ${ }^{1}$ for example. Many of these techniques have proven extremely useful in certain damage scenarios, but each technique is primarily a local technique. This means that these methods, in general, rely upon knowing where to look for damage and what to look for. Additionally, most of these techniques are impractical to apply to large-scale structures. Some of these issues were addressed with vibration-based techniques, where the structure is excited, either through ambient or applied loading, and the response observed. The practitioner then looks for some characteristic "feature" of the response which indicates the scope and possibly the location of the damage. Traditionally, most of these features have been derived from a modal analysis of the structure, e.g. resonant frequencies, mode shapes, flexibility, etc. ${ }^{2-5}$ Other non modal-based features have included cross-correlation, ${ }^{6}$ auto-regressive approaches, ${ }^{7}$ neural networks, ${ }^{8,9}$ time series dimensionality, ${ }^{10,11}$ and various other pattern recognition approaches. ${ }^{12,13}$ A good summary of these approaches may be found in. ${ }^{14}$ The implicit assumption, of course, is that damage somehow changes the dynamics of the structure and that the extracted feature(s) reflect this

Send correspondence to Michael Todd at email miketodd@ccs.nrl.navy.mil or phone (202) 767-1480. 


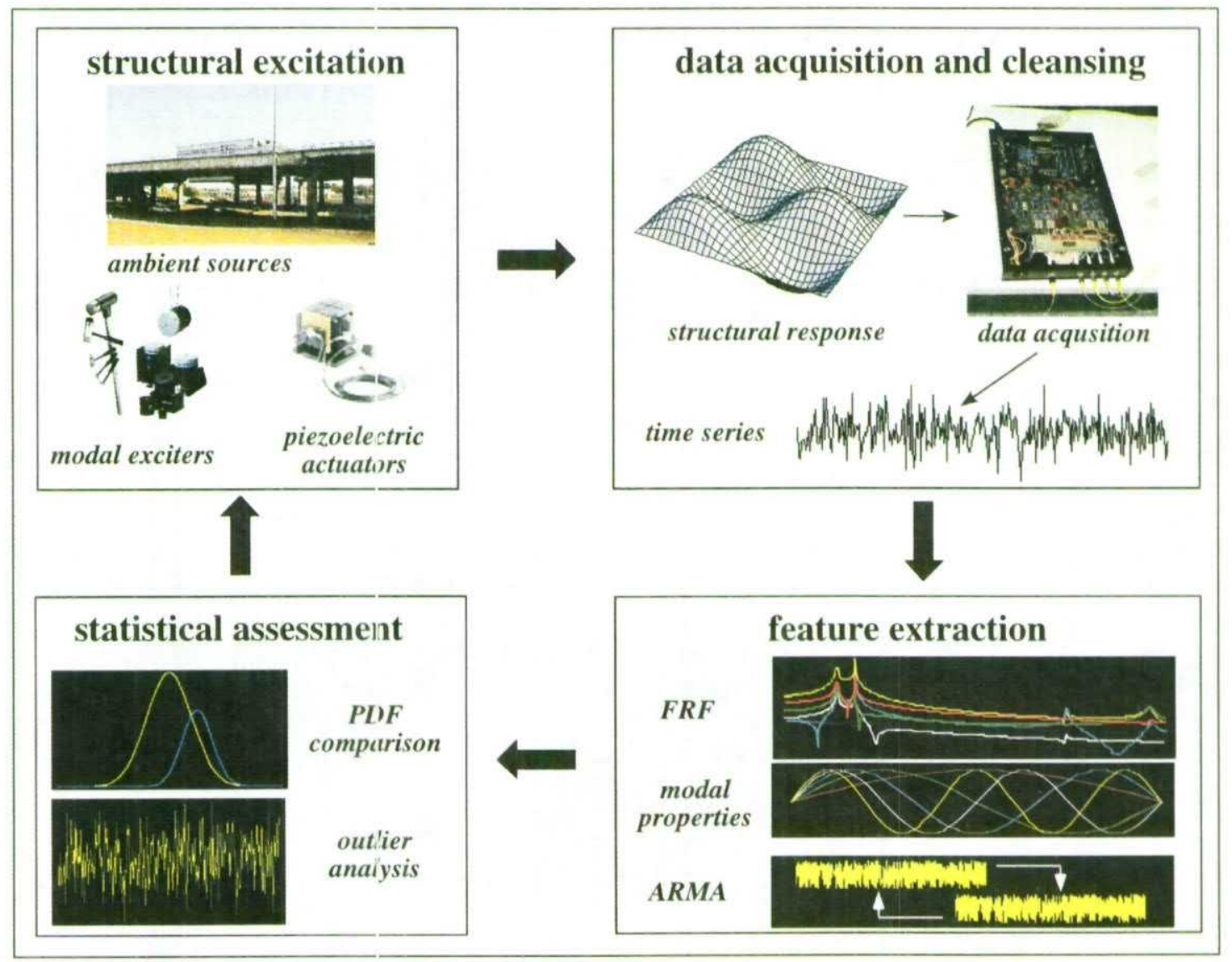

Figure 1: Vibration-based structural health monitoring paradigm.

change. More recently, research has introduced statistical tools to the problem, such as outlier detection ${ }^{15}$ discriminant analysis, ${ }^{16}$ and other classification schemes. ${ }^{17}$ With these advances, a modern vibration-based structural health monitoring paradigm could be described as in Figure 1: some form of excitation of the structural vibrations, measurement of the vibrations, extraction of appropriate features, and statistical assessment of the features for decision making.

Recently, Todd, Nichols, et al. ${ }^{18-20}$ have begun to propose methods based on imparting pre-determined deterministic dynamics upon the structure (i.e., not stochastic sources such as broadband noise) and extracting features based on the resulting steady-state dynamics (rather than properties based on the transient dynamics, such as modal properties). One issue central to this steady-state approach is finding a way to excite the structure such that the structure's steady-state response is low-dimensional enough to allow robust computation of features but high-dimensional enough that changes to the dynamics are observable. With a stochastic input, the steadystate response is too high dimensional to be easily exploited for calculating deterministic properties. The other excitation extreme may be a pure sinusoidal input, which results in a low-dimensional response but perhaps too low-dimensional, as the damage may manifest itself in a higher dimension. References ${ }^{18-20}$ showed how imparting a steady-state chaotic input to a structure can result in a low dimensional response whose steadystate properties may be exploited as sensitive indicators of damage. The approach takes advantage of the subtle ways in which the structure filters or alters a chaotic input in the presence of some evolving damage scenario and uses that information to develop empirical state space predictive models of the dynamics in a supervised learning mode context. 
This work will discuss using chaotic excitation as a structural interrogator and exploiting resulting attractor geometry in a diagnostic capacity. The approach is applied experimentally to an aluminum frame model building where damage is incurred by loosening selected bolted connections between members.

\section{PREDICTION ERROR AS A FEATURE}

An attractor is simply an invariant dynamic response to which initial condition trajectories eventually migrate under inevitable dissipation. For example, in a simple spring-mass-damper system subject to sinusoidal excitation, the (sole) attractor is a sinusoidal response at the same frequency as the excitation and some fixed phase difference. Eventually, under the damping, transient dynamics will dissipate to leave the steady-state response just described. Likewise, chaotic excitations of structures result in attractors, albeit more complex ones with often fractal rather than integral dimension properties. The goal in this current work is to take advantage of this low-dimensional steady-state chaotic response and develop a prediction-based approach. The idea is to use data taken from the undamaged "pristine" structure to reconstruct reference, or baseline, attractors. These data are to be compared to data collected from the structure at subsequent later times, when damage may (or may not) have accrued on the structure. Such an approach falls within the context of supervised learning in that known examples of data from each particular damage case must be obtained for later classification purposes. By using a simple prediction scheme, points on the "damaged" attractors are forecast using the baseline data as a model. Higher levels of damage will alter the structure's dynamic response causing these baseline models to lose their ability to make predictions. The error formed between prediction and measurement-prediction error-then becomes a good candidate feature for quantifying both the presence and magnitude of structural degradation. The models make no assumptions about the underlying system. This is in contrast to auto-regressive modeling techniques which implicitly assume a response coming from a structure for which a linear model is an accurate descriptor.

The method begins by recording a set of $N_{r}$ baseline time series ( $N_{r}$ independent experiments or runs) when the structure is in its (user-definable) baseline or reference condition. Recording $N_{r}$ such independent responses allows for the inclusion of ambient variation in the set of baseline data. Such variation is known to occur in practice and must be accounted for if damage-induced changes are to be distinguished from those due to environmental factors. We shall represent this baseline set of experiments by $X=\left\{x_{1}(n), x_{2}(n), \cdots, x_{N_{r}}(n)\right\}$ where each $x_{u}(n), u=1 \cdots N_{r}$, is a vector of discretely sampled values of structural response (time series) consisting of $n=1 \cdots N$ points. The discrete time index " $n$ " simply refers to the value recorded at sample time $t_{s}=n \Delta t$ where $1 / \Delta t$ is the sampling frequency. Similarly, at each damage case a set of data $Y_{k}=$ $\left\{y_{1 k}(n), y_{2 k}(n), \cdots, y_{N_{r} k}(n)\right\}, k=1 \cdots N_{d}$ is recorded, where $N_{d}$ is the number of damage scenarios to consider and the $y_{u^{\prime} k}, u^{\prime}=1 \cdots N_{r}$, are response time series recorded from the structure at damage case " $k$ " in the same fashion that the set $X$ was created. The next step is to use the baseline data $X$ to empirically generate attractor-based models of the pristine structure's dynamics and observe the degree to which they predict the dynamics of the $N_{d}$ damaged data sets.

The algorithm used here is adopted from Schreiber, ${ }^{21}$ where it was used to detect non-stationarity in timeseries data. A simple attractor-based prediction scheme is used on the undamaged data to forecast the values of the damaged data some number of time steps $s$ into the future. Each of the baseline time series may be used to reconstruct a set of baseline attractors $\mathbf{x}_{u}(n), u=1 \cdots N_{r}$, using the embedding theorem, ${ }^{22}$ where we have now adopted boldface type to indicate an attractor rather than a single time series. Similarly, each of the "damaged" data sets $Y_{k}$ will yield $N_{r}$ "damaged" attractors, denoted $\mathbf{y}_{u^{\prime} k}(n), u^{\prime}=1 \cdots N_{r}, k=1 \cdots N_{d}$. The method proceeds by selecting a specific baseline attractor $u$ and the attractor whose dynamics are being tested, $u^{\prime}$ at a given damage case $k$. The subscripts $\left(u, u^{\prime}, k\right)$ are therefore dropped in the following discussion for clarity. Given a randomly selected trajectory with time index $f$ on a reconstructed "damaged" attractor, $\mathbf{y}(f)$, the algorithm selects the set of points on the baseline attractor $\mathbf{x}(n)$ that are within some radius $\epsilon$ of that trajectory

$$
U_{\epsilon}^{\mathbf{x}(p)}(\mathbf{y}(f))=\mathbf{x}(p):\|\mathbf{x}(p)-\mathbf{y}(f)\|<\epsilon .
$$

where $\|\cdot\|$ denotes the vector norm. No temporal relationship need exist between the indices $p$ and $f$, as the set is constructed purely by geometry; in other words, the $p$ values of $\mathbf{x}(n)$ are not selected explicitly by the 
algorithm, but rather through the Euclidean distance of the specific point from the fiducial point is what is selected, with the time index of that point passively carried along for bookkeeping. This is in contrast to an autoregressive approach, which is based on temporal relationships. The idea here is to describe the evolution of the neighborhood $U_{\epsilon}^{\mathbf{x}(p)}(\mathbf{y}(f))$ and to use this description as a predictor for how subsequent data should behave. The predicted value for $\mathbf{y}(f)$ at $s$ time steps into the future, denoted $\hat{\mathbf{y}}(f+s)$, becomes

$$
\hat{\mathbf{y}}(f+s)=\frac{1}{\left|U_{\epsilon}^{\mathbf{x}(p)}(\mathbf{y}(f))\right|} \sum_{\mathbf{x}(p) \in U_{\epsilon}^{\mathbf{x}(p)}(\mathbf{y}(f))} \mathbf{x}(p+s),
$$

where the quantity $\left|U_{\epsilon}^{\mathrm{x}(p)}(\mathbf{y}(f))\right|$ simply denotes the number of points in the neighborhood. The predicted value is the average of predicted values for the neighborhood. In this sense the baseline attractors are used as "lookup" tables which contain the various patterns present in the data. The working hypothesis is that these tables will lose their ability to serve as an accurate database as the dynamics are altered by damage. The prediction horizon $s$ will depend on the rate at which the data are acquired and the specific application. For health monitoring purposes, assuming reasonably sampled data, $s=1$ will suffice. While more complicated prediction schemes exist, this is among the simiplest models one can use to quantify the evolution of the dynamics. Because we only seek to distinguish one attractor from another, the quality of the predictions is of diminished importance and the simplest, most computationally efficient scheme is considered optimal. Variations of this algorithm have been used for prediction and data cleansing. ${ }^{23,24}$

Once the predictions has been made the $s$-step prediction error for trajectory $f$ is quantified by

$$
\gamma=\frac{1}{N} \sum_{f=1}^{N}(\hat{\mathbf{y}}(f+s)-\mathbf{y}(f+s))^{2},
$$

This quantity is then normalized by the variance, $\sigma^{2}$, of the baseline signal

$$
\hat{\gamma}=\frac{\gamma}{\sigma^{2}}
$$

to yield the normalized nonlinear cross-prediction error for time index $f$. The process is repeated for some randomly selected subset of the total number of points on the attractor $M: M<N$ resulting in a vector of prediction errors $\hat{\gamma}_{i}, i=1 \cdots M$, for each pair of attractors under consideration, $\left(u, u^{\prime}\right)$. However, because there are $N_{r}$ attractors with which to make comparisons $\left(u, u^{\prime}=1 \cdots N_{r}\right)$, there are actually $N_{m}=M N_{r}^{2}$ total predictions at a given damage case. The entire feature vector at damage case $k$ is therefore denoted $\hat{\gamma}_{i k}, i=1 \cdots N_{m}, k=1 \cdots N_{d}$.

In computing this feature, one must also compute the auto-prediction error by replacing $\mathbf{y}(f)$ with $\mathbf{x}(f)$ in Equation (1). The resulting $\hat{\gamma}_{i 1}$ gives some idea of the prediction error one would expect to find in the instance that the dynamics are not changing. However, in order to make such a comparison we must add the constraint that comparisons between attractors $\left(u, u^{\prime}\right)$ be made such that $u<u^{\prime}$. The problem is that comparing $\mathbf{x}_{1}$ and $\mathbf{x}_{2}$, for example, followed by a cornparison of $\mathbf{x}_{2}$ with $\mathbf{x}_{1}$ could potentially bias the data and therefore such duplicate pairings should be eliminated from consideration. Although comparisons between data from different damage scenarios do not suffer this effect (each baseline attractor $\mathbf{x}$ could be used to predict each damaged attractor $\mathbf{y}$ ), the constraint is majntained for consistency so that at each damage case the total number of predictions is reduced to $N_{m}=M N_{r}\left(N_{r}-1\right) / 2$ values, as opposed to $N_{m}=M N_{r}^{2}$ if duplicate pairs been been included.

One other consideration is the possibility of obtaining data from multiple locations on a structure. While the preceding discussion assumes data collected at a single location, many health monitoring studies incorporate data from many spatial locations. Assuming structural response data have been collected from sensors at $N_{s}$ different locations, the final set of features is denoted $\hat{\gamma}_{i j k}$ where $i=1 \cdots N_{m}$ is the member index for an individual prediction, $j=1 \cdots N_{s}$ spans the range of sensors, and $k=1 \cdots N_{d}$ remains the damage index. It is 


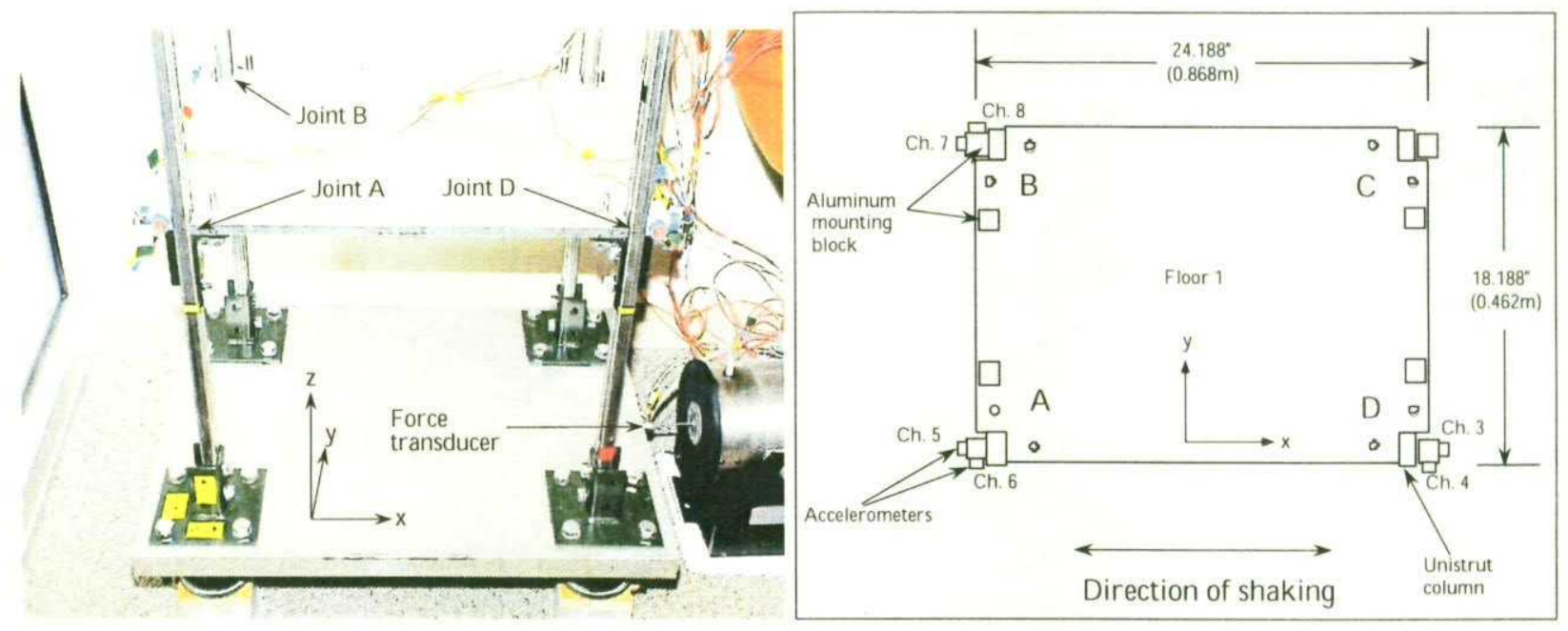

Figure 2: Experimental set-up showing instrumented Floor 1 at Joints A, B, and D.

convenient to define the final collection of features by the set

$$
\mathbf{S}_{i}=\left\{\begin{array}{cccc}
\hat{\gamma}_{i 11} & \hat{\gamma}_{i 12} & \cdots & \hat{\gamma}_{i 1 N_{d}} \\
\hat{\gamma}_{i 21} & \hat{\gamma}_{i 22} & \cdots & \hat{\gamma}_{i 2 N_{d}} \\
\vdots & \vdots & \ddots & \vdots \\
\hat{\gamma}_{i N_{o} 1} & \hat{\gamma}_{i N_{o} 2} & \cdots & \hat{\gamma}_{i N_{s} N_{d}}
\end{array}\right\},
$$

in which each $\mathbf{S}_{i}\left(i=1 \cdots N_{m}\right)$ is a matrix of observations of prediction error extracted from data recorded at sensor location $j$ and structural condition $k$. We now turn our attention to the task of using the $N_{m}$ observations at each sensor location to classify the data according to damage.

\section{EXPERIMENT}

This approach was tested by exciting a scaled three-story frame structure with a chaotic Lorenz oscillator, such that the structural system model could be written

$$
\begin{aligned}
\dot{z}_{1} & =16\left(z_{2}-z_{1}\right) \\
\dot{z}_{2} & =40 z_{1}-z_{2}+z_{1} z_{3} \\
\dot{z}_{3} & =-4 z_{3}+z_{1} z_{2} \\
\dot{\mathbf{x}} & =\mathbf{A x}+\mathbf{B z},
\end{aligned}
$$

where the first three equations describe the Lorenz oscillator in a chaotic regime, and the last equation is the state equation of the structure coupled to the oscillator through $\mathbf{B}$.

The test structure, constructed of aluminum Unistrut columns and aluminum floor plates, is shown at the top of Figure 2. The floors were 1.3-cm-thick (0.5-in-thick) aluminum plates with two bolt connections to brackets on the Unistrut columns. The base was a 3.8-cm-thick (1.5-in-thick) aluminum plate. Support brackets for the columns were bolted to this plate. A further discussion of this structure may be found in. ${ }^{25}$ An electromechanical shaker was connected to the structure at the mid-height of the base plate so that translational motion could be imparted.

The structure was instrumented with 6 piezoelectric accelerometers on Floor 1 only (bottom of Figure 2). Accelerometers were mounted on blocks glued to three of the Unistrut columns (Joints A, B and D) at the floor 
level, in both in-plane directions. Additionally, a force transducer was mounted between the stinger and the base plate. This force transducer was used to measure the input to the base of the structure. A commercial data acquisition system controlled from a laptop PC was used to digitize the accelerometer and force transducer analog signals.

\begin{tabular}{|c|c|}
\hline Damage Case & Damage Location(s) \\
\hline \hline 0 (baseline) & all bolts at 12.4 Nm (110 in-lbf) \\
\hline 1 & Joint D only bolts removed \\
\hline 2 & Joint A only bolts removed \\
\hline 3 & Joint B only bolts removed \\
\hline 4 & Joints A and D bolts removed \\
\hline 5 & Joints A, B, and D bolts removed \\
\hline
\end{tabular}

Table 1: Description of damage cases.

A simple input/output MATLAB SIMULINK ${ }^{\mathrm{TM}}$ model along with XPC Target ${ }^{\mathrm{TM}}$ and a commercially available signal conditioner were used to send the pre-digitized chaotic Lorenz waveform to the shaker. The waveform was sent at a $1000 \mathrm{~Hz}$ shaker update rate, and the analog sensors were sampled at $370.3 \mathrm{~Hz}$. Five sets of baseline undamaged data sets were recorded before damage was introduced to the structure. Damage was introduced by removing two bolts at various combinations of the connections between Floor 1 and the three Unistruts. The baseline condition and five damage scenarios are summarized in Table 1 . The test procedure was repeated at each of the damage cases, resulting in $N_{r}=5$ runs at each of $N_{d}=6$ total damage cases (including the baseline) for each of the $N_{s}=6$ accelerometers ( 5 runs $\times 6$ sensors $\times 6$ damage cases $=180$ separate data sets).
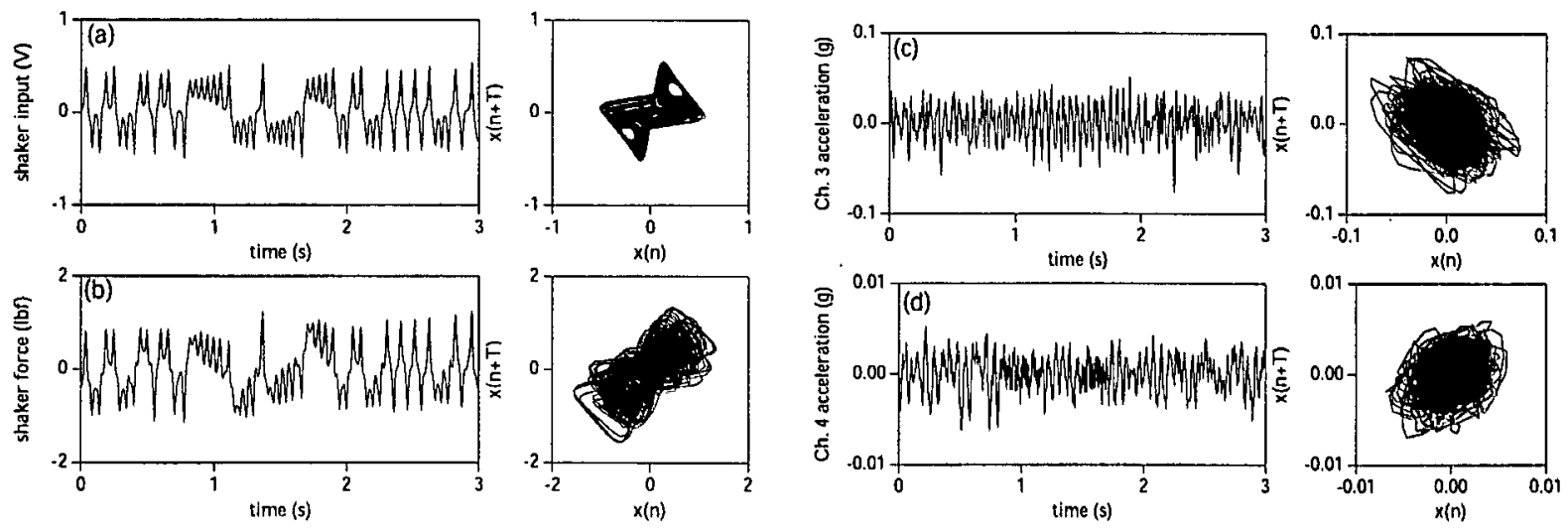

Figure 3. Typical three-second time windows (left column) and attractors (right column) for (a) raw voltage input to shaker, (b) force transducer output, (c) Ch. 3 acceleration, and (d) Ch. 4 acceleration.

\section{RESULTS}

\subsection{Individual Sensor Results}

Representative shaker input (pre-digitized Lorenz waveform), force transducer response, and acceleration response signals are shown in Figure 3, along with their corresponding reconstructed attractors. The driving signal and the force felt by the structure exhibit the low-dimensional features characteristic of chaotic waveforms. The force time series very closely tracks the input Lorenz waveform, with some differences primarily due 

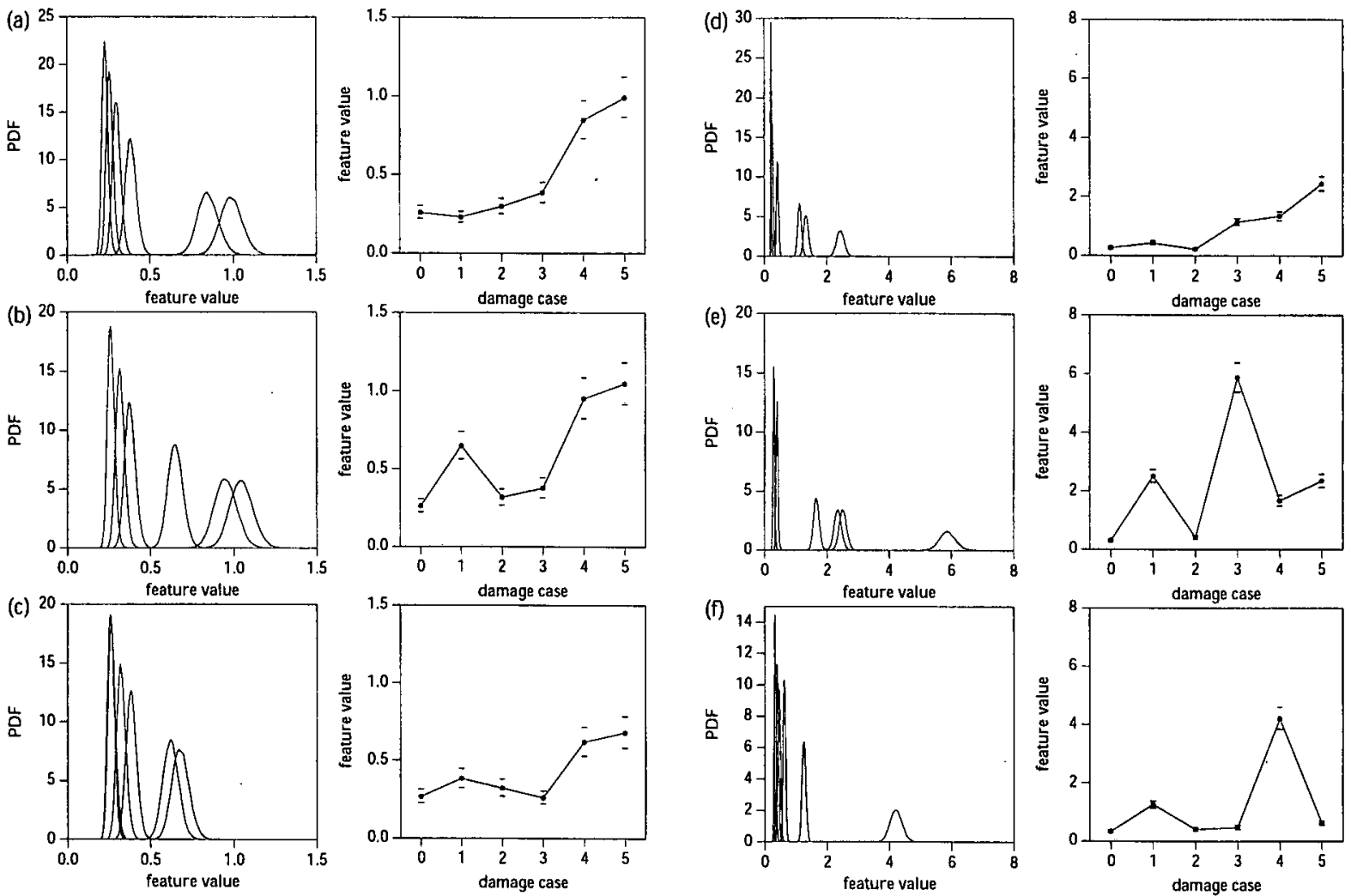

Figure 4. Probability density function estimates, mean values, and $95 \%$ quantile bounds for $\mathrm{Ch} .3$ (a), Ch. 5 (b), Ch. 7 (c), Ch. 4 (d), Ch. 6 (e), and Ch. 8 (f).

to structural feedback at the transducer and possibly even stinger dynamics. Response acceleration time series, however, are clearly less structured and illustrate the filtering effect of the structure on the input signal. Both co-directional (Ch. 3) and contra-directional (Ch. 4) sensor data are shown in order to highlight the differences in response data from these two directions. In this context, "co-directional" means in the same direction as the excitation, while "contra-directional" means perpendicular to that direction. Co-directional acceleration data are greater in magnitude than contra-directional data, as expected. The contra-directional data appear to have some higher frequency content, resulting in a more "tightly wound" attractor. This does not pose a challenge from a modeling perspective, as predictions made on data coming from a given sensor are made using only the baseline data from that same sensor.

For each undamaged/damaged attractor pair, $M=3,276$ individual predictions were made using the undamaged attractor as the baseline. This results in $N_{m}=3,276 \times 5(5-1) / 2=32,760$ values of prediction error per damage case per sensor which will reflect any ambient variation present in the 5 independent runs under each damage case. Each of the feature vectors in the set $\mathbf{S}_{i} i=1 \cdots N_{m}$ were resampled ${ }^{26}$ into $\beta=5,000$ clusters of size $\mathcal{C}=1,000$. Figure 4 summarizes the behavior of the prediction error feature for data from co-directional sensor channels 3,5 , and 7 (a-c) and contra-directional channels 4,6 , and 8 (d-f). The left-hand plot in each sub-figure shows the probability density function (PDF) estimate for all the data, which appear quite Gaussian due to the resampling procedure. From these distributions, we obtained $N_{s}$ means and $95 \%$ quantile limits for each of the $N_{d}$ damage cases. These limits are plotted beside their corresponding PDFs in order to better illustrate the discriminating power of the data from a single sensor.

In general there is very reasonable separation among the PDFs for both co- and contra-directional sensors, 
implying a good ability to distinguish among the various damage cases. This is reflected in the control limits which show no overlap for many cases. Features obtained from the co-directional data can successfully discriminate among many damage cases. Data collected from channel 3 can clearly distinguish cases 3,4 , and 5 from case 0 (undamaged), although it should be noted that cases 4 and 5 cannot be distinguished from each other, a problem to be addressed shortly using multivariate analysis. Channels 5 and 7 appear sensitive to damage cases 1,4 and 5 . The fact that all sensors tended to produce high prediction errors for damage levels 4 and 5 should not seem surprising. The last two damage cases were the most severe in terms of the degree to which the structure's connectivity was degraded, affecting the dynamics in such a way as to be easily observed by the co-directional sensors. Features extracted from the contra-directional data produced much more interesting results. In general the separation bietween PDFs is larger than for co-directional data, as certain damage cases become more clearly highlighted. Channel 6 , for example, highlights cases 1 and 3 more so than does channel 5 (although the trend is nearly the same). Furthermore, damage cases 4 and 5 do not always produce the largest mean prediction error implying that the dynamics recorded from the contra-directional sensors are responding to degradation differently than their co-directional counterparts (see plots from channels 6 and 8).

As a general rule, the contra-directional sensors overall performed better at discriminating among damage cases. This likely is explained by investigating a close-up of any particular damage case. The bolts removed in each damage case were aligned with the co-directional axis. Such a removal may allow some impact-like motion in that direction, but most of the time, the structure remains constrained. However, in the contra-direction, all bolt-induced stiffness has been removed, and relative joint dynamics are easily excited, even though they are perpendicular to the plane of forcing. The slightest asymmetry (which necessarily exists) excites contradirectional dynamics, and these dynamics are suppressed with bolts in place.

\subsection{Multivariate Data Discrimination}

Although the single sensor data is useful, it falls short of the end goal which is to classify data according to the 6 damage cases. To this end a multivariate linear discriminant analysis procedure ${ }^{27,28}$ was employed in order to take advantage of the fact that we have multiple sources of data. Multivariate discriminant analysis seeks the linear combination of data which minimizes the within group variance, that is differences between linear combinations of sensor data at within single damage case, while at the same time maximizing differences between data collected at different damage levels. Following the supervised learning paradigm, the sets of prediction error for each sensor were split into two halves $\mathbf{S}_{i} \rightarrow \mathbf{S}_{i}^{(1)} i=1 \cdots N_{m} / 2, \mathbf{S}_{i}^{(2)} \quad i=N_{m} / 2+1 \cdots N_{m}$, each containing $N_{m} / 2$ members. The first half of the data, $\mathbf{S}_{i}^{(1)}$, were resampled, again using $\beta=5,000 \mathcal{C}=1,000$, and then used to find the discriminant functions, i.e., to "train" the discriminator. The second half of the data $\left(\mathbf{S}_{i}^{(2)}\right.$ were then used to assess the strength of the classification scheme. To this end, $\beta=30,000$ sample means were drawn from the resampled data of $\mathbf{S}^{(2)}$ for each damage scenario and multiplied by the discriminator. This constitutes a "blind" study ir. which data coming from a structure of "unknown" condition are classified according to pre-defined damage levels. In the ideal case, the transformed data will fall into 6 independent intervals, each corresponding to a different damage scenario. The results are shown in Figure 5 . The PDFs for the 6 sensors are shown as the smaller plots labeled Case $0 \rightarrow$ Case 5 corresponding to the 6 damage scenarios. The center plot shows the PDFs for the transformed data, i.e., after application of the discriminator.

The tightly-packed PDFs from the individual sensors are combined in such a way that 6 independent intervals are indeed created. In fact, all damage scenarios appear to be classified as independent distributions with the exception of possibly cases 0 and 2 . In order to quantify the discriminating power of the technique, each of the classified points were compared to the actual damage case from which the sampled data were taken. The percentage of the data that were classified correctly are shown in the confusion matrix, Table 2 . These percentages were obtained by examining the degree of overlap in the distributions for each pair of damage scenarios. Distributions that are completely separate from one another result in $100 \%$ classification while varying degrees of overlap result in diminished success rates.

The data appear to be projected into separate damage "bins" with near $100 \%$ accuracy. A success rate of $100 \%$ is achieved for all classifications except between damage cases 0 and 2 . In this case there is overlap between the resulting distributions (seen in the main plot of Figure 5) such that only a $86 \%$ rate is achieved 


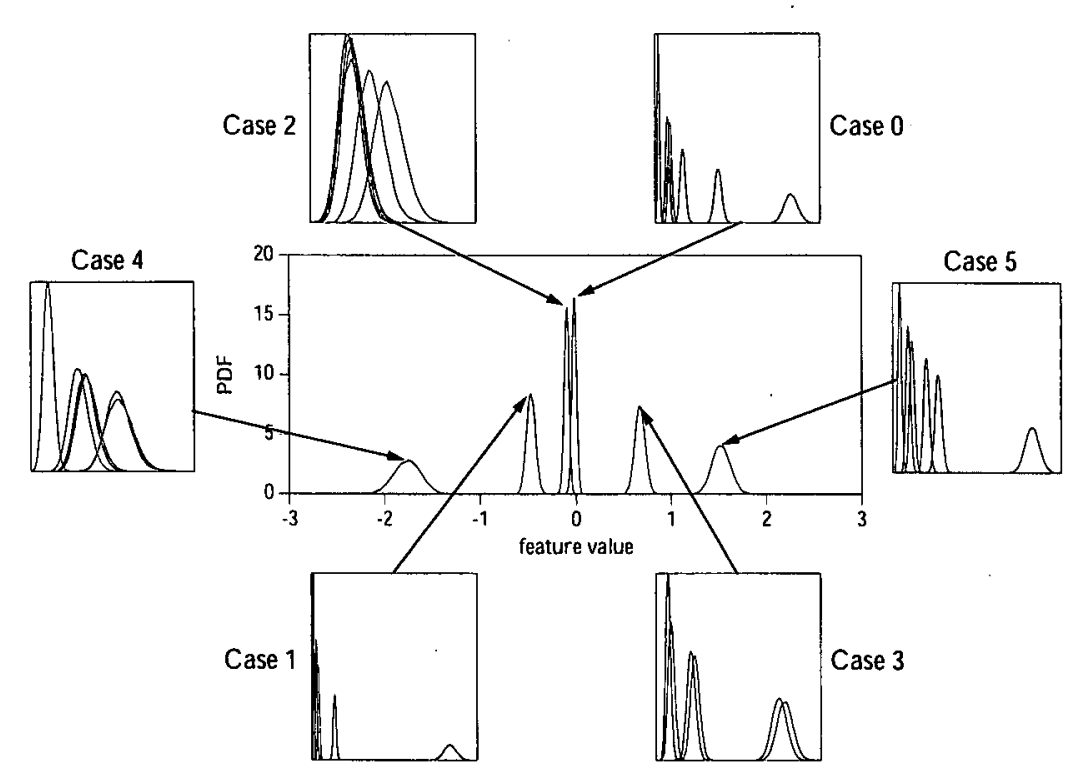

Figure 5: Optimized damage classification using linear discriminant analysis.

\begin{tabular}{|c|c|c|c|c|c|}
\hline Case & 1 & 2 & 3 & 4 & 5 \\
\hline 0 & 100.0 & 86.0 & 100.0 & 100.0 & 100.0 \\
1 & $\times$ & 100.0 & 100.0 & 100.0 & 100.0 \\
2 & $\times$ & $\times$ & 100.0 & 100.0 & 100.0 \\
3 & $\times$ & $\times$ & $\times$ & 100.0 & 100.0 \\
4 & $\times$ & $\times$ & $\times$ & $\times$ & 100.0 \\
\hline
\end{tabular}

Table 2: Confusion matrix for "blind" study using linear discriminant analysis.

for discriminating between these two cases. In no cases did the supposedly optimized linear discriminant analysis fail to perform better than any given individual sensor. If that had been the case, that could imply that somenonlinear combination of the sensor data may be required for accurate results. Exploiting nonlinear discriminators will be the goal of future work.

\section{CONCLUSIONS}

This work has illustrated the value of a steady-state approach to vibration-based structural health monitoring. It is possible, through the use of chaotic excitation, to generate a deterministic structural response which may be used to build empirical models of the system's dynamics. Deviations in these models will cause their predictive power to diminish and thus provide a useful means to detect damage. The power of this approach is that it directly searches for changes in the dynamics. This is in contrast to the more traditional "transient" approaches which proceed by extracting features which may or may not be sensitive to damage induced changes to the structure's response.

Using linear discriminant analysis clearly shows that incorporating multiple sensor data in the analysis is advantageous, as it significantly increases the discriminating power of the data. Multivariate discriminant analysis will likely play a large role in future studies which fall under supervised learning approaches. The procedure is optimal in the sense that it can take spatially extended data and maximize the discriminating power of that data in the presence of ambient variation. The combination of a steady-state, model-based 
approach can yield powerful results. We have shown that this approach is extremely effective in discriminating between the various damage cases on an experimental aluminum frame structure.

\section{ACKNOWLEDGMENTS}

The authors acknowledge the National Research Council (NRC) for funding a post-doctoral fellowship for the third author and acknowledge both the U.S. Naval Research Laboratory and Los Alamos National Laboratory for jointly supporting this work.

\section{REFERENCES}

1. J. E. Doherty, "Nondestructive evaluation," in Handbook on Experimental Mechanics, A. S. Kobayashi, ed., ch. 12, pp. 527-557, VCH Publishers, Inc., New York, 1993.

2. C. R. Farrar, T. A. Duffey, S. W. Doebling, and D. A. Nix, "A statistical pattern recognition paradigm for vibration-based structural health monitoring," in Structural Health Monitoring 2000, pp. 764-773, 1999.

3. A. K. Pandey and M. Biswas, "Damage detection in structures using changes in flexibility," Journal of Sound and Vibration 169, pp. 3-17, 1994.

4. R. P. Sampaio, N. M. M. Maia, and J. M. M. Silva, "Damage detection using the frequency-responsefunction curvature method," Journal of Sound and Vibration 226(6), pp. 1029-1042, 1999.

5. P. Verboven, E. Parloo, P. Guillaume, and M. V. Overmeire, "Autonomous structural health monitoring - part i: Modal parameter estimation and tracking," Mechanical Systems and Signal Processing 16(4), pp. $637-657,2002$.

6. A. C. Okafor, K. Chandrashekhara, and Y. P. Jiang, "Location of impact in composite plates using waveform-based acoustic emission and gaussian cross-correlation techniques," in Proc. SPIE 2718, pp. 291$302,1996$.

7. H. Sohn, C. R. Farrar, N. F. Hunter, and K. Worden, "Structural health monitoring using statistical pattern recognition techniques," Jourrial of Dynamic Systems, Measurement, and Control 123, pp. 706-711, 2001.

8. C. M. Bishop, "Novelty detection and neural network validation," in IEE Proceedings: Vision, Image, and Spectral Processing, 141, 1994.

9. Y. Q. Ni, B. S. Wang, and J. M. Ko, "Selection of input vectors to neural networks for structural damage identification," in Proc. SPIE 3671, pp. 270-280, 1999.

10. D. Logan and J. Mathew, "Using the correlation dimension for vibration fault diagnosis of rolling element bearings - i. basic concepts," Mechanical Systems and Signal Processing 10(3), pp. 241-250, 1996.

11. D. Logan and J. Mathew, "Using the correlation dimension for vibration fault diagnosis of rolling element bearings - ii. selection of experimental parameters," Mechanical Systems and Signal Processing 10(3), pp. 251-264, 1996.

12. D. George, N. Hunter, C. Faurar, and R. Deen, "Identifying damage sensitive features using nonlinear time series and and bispectral analysis," in Proc. of IMAC XVIII: A Conference on Structural Dynamics, pp. $1796-1802,2000$.

13. K. Worden and A. J. Lane, "Damage identification using support vector machines," Journal of Smart Materials and Structures 10, Fp. 540-547, 2001.

14. S. W. Doebling, C. R. Farrar, and M. B. Prime, "A summary review of vibration-based identification mehods," Shock and Vibration Digest 205(5), pp. 631-645, 1998.

15. K. Worden, "Structural fault detection using a novelty measure," Journal of Sound and Vibration 201, pp. 85-101, 1997.

16. M. L. Fugate, H. Sohn, and I. R. Farrar, "Vibration based damage detection using statistical process control," Mechanical Systems and Signal Processing 15, pp. 707-721, 2001.

17. I. Trendafilova and H. V. Brussel, "Non-linear dynamics tools for the motion analysis and condition monitoring of robot joints," Mechanical Systems and Signal Processing 15, pp. 1141-1164, 2001.

18. M. D. Todd, J. M. Nichols, and L. N. Virgin, "Vibration-based damage assessment utilizing state space geometry changes: Local attractor variance ratio," Smart Structures and Materials 10(5), pp. 1000-1008, 2001. 
19. J. M. Nichols, M. D. Todd, S. T. Trickey, and L. N. Virgin, "Structural health monitoring through chaotic interrogation," Meccanica (in press) , 2001.

20. L. N. V. J. M. Nichols, M. D. Todd and J. Nichols, "On the use of attractor dimension as a feature in structural health monitoring," Mechanical Systems and Signal Processing, 2002.

21. T. Schreiber, "Detecting and analyzing nonstationarity in a time series using nonlinear cross predictions," Physical Review Letters 78(5), pp. 843-846, 1997.

22. F. Takens, "Detecting strange attractors in turbulence," in Dynamical Systems and Turbulence, D. Rand and L.-S. Young, eds., Lecture Notes in Mathematics 898, pp. 366-81, Springer-Verlag, New York, 1981.

23. H. Kantz and T. Schreiber, Nonlinear Time Series Analysis, Cambridge University Press, 1999.

24. J. M. Nichols and J. D. Nichols, "Attractor reconstruction for nonlinear systems: A methodological note," Mathematical Biosciences 171, pp. 21-32, 2001.

25. T. R. Fasel, S. Gregg, T. Johnson, C. R. Farrar, and H. Sohn, "Experimental modal analysis and damage detection in a simulated three story building," in Proceedings of IMAC XX: A Conference on Structural Dynamics, Society of Experimental Mechanics, 2002.

26. B. F. Manly, Randomization and Monte Carlo Methods in Biology, Chapman and Hall, 1991.

27. L. Lebart, A. Morineau, and K. M. Warwick, Multivariate Descriptive Statistical Analysis, John Wiley \& Sons, Inc., 1984.

28. W. W. Cooley and P. R. Lohnes, Multivariate Data Analysis, John Wiley \& Sons, Inc., 1971. 